\title{
Correction to: Antibody against TDP-43 phosphorylated at serine 369 suggests conformational differences of TDP-43 aggregates among FTLD-TDP subtypes
}

\author{
Manuela Neumann ${ }^{1,2}$ (D) Petra Frick ${ }^{1} \cdot$ Francesca Paron $^{3} \cdot$ Jonas Kosten $^{1} \cdot$ Emanuele Buratti $^{3} \cdot$ Ian R. Mackenzie $^{4}$
}

Published online: 3 November 2020

(c) Springer-Verlag GmbH Germany, part of Springer Nature 2020

\section{Correction to: Acta Neuropathologica (2020) 140:645-658 https://doi.org/10.1007/s00401-020-02207-w}

Due to a mistake with incorrect assignment of the originally planned amino acid residues 368-379 to the protein sequence of TDP-43 for antibody production by the contracted company, a peptide corresponding to amino acids 362-373 with a phosphorylated serin at 369 instead of a peptide corresponding to amino acids $368-379$ with a phosphorylated serin at 375 was synthesized and used for antibody generation and purification.

Thus, the study in fact describes findings with a novel antibody against TDP-43 phosphorylated at serine 369 and not against TDP-43 phosphorylated at serine 375 .

All presented results and conclusions of the study with the main finding of TDP-43 type-specific staining properties of this antibody are not affected.

1. The title should read as follows:
Antibody against TDP-43 phosphorylated at serine 369 suggests conformational differences of TDP-43 aggregates among FTLD-TDP subtypes.

2. Throughout the text, figures and table 2 all references to pTDP- $43^{\mathrm{S} 375}$ should actually refer to pTDP- $43^{\mathrm{S} 369}$.

3. The sentences in the "Methods" section should read as follows:

Generation of polyclonal pTDP-43 3369 antibody

The rabbit polyclonal antibody against TDP-43 phosphorylated at serine 369 was produced by Biosense/Eurogentec using their custom polyclonal antibody cross-affinity purification service. Briefly, rabbits were immunized with a phosphopeptide corresponding to amino acid 362-373 of human TDP-43: ac-EPNQAFGS(p)GNNS-C-CONH 2 . Phospho-specific antibodies were purified using affinity chromatography with modified and unmodified peptides.

Publisher's Note Springer Nature remains neutral with regard to jurisdictional claims in published maps and institutional affiliations.
The original article can be found online at https://doi.org/10.1007/ s00401-020-02207-w.

Manuela Neumann

manuela.neumann@dzne.de

1 Molecular Neuropathology of Neurodegenerative Diseases, German Center for Neurodegenerative Diseases (DZNE) Tübingen, Otfried-Müllerstr. 23, 72072 Tübingen, Germany

2 Department of Neuropathology, University Hospital of Tübingen, Tübingen, Germany

3 Department of Molecular Pathology, International Centre for Genetic Engineering and Biotechnology (ICGEB), Trieste, Italy

4 Department of Pathology, University of British Columbia and Vancouver General Hospital, Vancouver, Canada 